\title{
The Effectiveness of Energy Feedback for Conservation and Peak Demand: A Literature Review
}

\author{
Desley Vine, Laurie Buys, Peter Morris \\ Science \& Engineering Faculty, Queensland University of Technology, Brisbane, Australia \\ Email: d.vine@qut.edu.au
}

Received January 10, 2013; revised February 6, 2013; accepted March 4, 2013

\begin{abstract}
This paper reviews electricity consumption feedback literature to explore the potential of electricity feedback to affect residential consumers' electricity usage patterns. The review highlights a substantial amount of literature covering the debate over the effectiveness of different feedback criteria to residential customer acceptance and overall conservation and peak demand reduction. Researchers studying the effects of feedback on everyday energy use have observed substantial variation in effect size, both within and between studies. Although researchers still continue to question the types of feedback that are most effective in encouraging conservation and peak load reduction, some trends have emerged. These include that feedback be received as quickly as possible to the time of consumption; be related to a standard; be clear and meaningful and where possible both direct and indirect feedback be customised to the customer. In general, the literature finds that feedback can reduce electricity consumption in homes by 5 to 20 percent, but that significant gaps remain in our knowledge of the effectiveness and cost benefit of feedback.
\end{abstract}

Keywords: Electricity; Energy Conservation; Feedback; Residential

\section{Introduction}

The only feedback received by many Australian households on their electricity consumption is their quarterly electricity bill [1]. The information (energy consumption per tariff, a comparison of consumption from previous billing periods and total owed for the current billing cycle) and the frequency of the Australian electricity bill have changed little in several decades [1]. This largely un-itemised, non-visual and infrequent feedback on their electricity consumption has been likened to driving cars without any information on the volume or price of fuel consumed and instead receiving a non-itemised invoice at some time in the future for the combined fuel consumption of all family vehicles [2]. This lack of information has become increasingly problematic in Australia, given forecasts for the price of domestic electricity increasing by over 37\% between 2010.11 and 2013.14 [3]. This paper reviews electricity consumption feedback literature to explore the potential of electricity feedback to affect residential consumers' electricity usage patterns. "Feedback" in this context is household-specific information on electricity use.

There has been regular enquiry in the literature to improve electricity feedback to consumers since the 1970s [4]. Various methods of providing feedback have been explored including more detailed electricity bills [5], self-reading of meters [6], interactive tools [7] and in- home displays featuring various data including consumption comparisons and visualisations [2,8,9]. Feedback is also regularly studied in conjunction with additional instruments for electricity-saving or behaviour change such as time-of-use or real-time pricing [10] and critical peak pricing [2]. Encouraging the provision of feedback through subsidies, mandates, or other policies could be part of future utility demand-side management (DSM) programs [11]. However, consistent throughout the majority of feedback literature is the finding that feedback is linked to a conservation effect [12].

\section{Functions of Feedback}

Policies that provide feedback after consumption can be either "direct" or "indirect" [13]. Direct or real-time feedback is immediate and from a meter or other display monitor and has been found to provide greater energy savings than indirect feedback methods [14] which is information that has been processed in some way, e.g. more detailed electricity bills or household-specific advice for reducing electricity use $[10,13]$. Real-time or direct feedback has benefits over enhanced feedback. First, it can impact habitual, repetitive behaviour such as turning off lights or unplugging appliances $[15,16]$. People perform many everyday activities without reflection according to routines developed over time and this includes use of electricity [12]. Economists believe that full 
disclosure of information creates rational consumers [17]. With complete information people act rationally with the objective of maximising utility for dollars spent [18]. Without complete information, it is argued that people are imperfectly rational [17]. Direct feedback then should enhance other demand-response and DSM programs, including making users more responsive to real-time or time-of-use pricing programs and realising the conesquent benefits of load-shifting [2,11] thereby affecting peak consumption.

The second major benefit of direct feedback is the effect it can have on appliance purchasing decisions, as consumers notice from feedback that certain appliances are heavy energy consumers, they can consider replacing them with more efficient ones [12]. It has been argued that this could also lead to behavioural adjustment [16] with for example, people upon learning the real cost of leaving their television switched on decide to switch it off when no-one is in the room. Behavioural adjustment or product choice is more relevant when costs and the extent of energy use are made clearly apparent to the consumer [19]. The third major benefit is that real-time feedback can be more easily customised for individual households $[15,20]$. With the proper software to manipulate data, feedback could present usage patterns in formats most helpful to individual households [20,21]. Reviews of direct feedback experiments suggest direct feedback interventions yield between $5 \%$ and $15 \%$ energy savings for the time that they are installed, however their lasting impacts on behaviour are much less certain [2,13,22]. For example, a 15 month study undertaken by van Dam and colleagues [23] found that initial savings of $7.8 \%$ after four months could not be sustained in the medium to longer term.

\section{Empirical Studies}

Researchers studying the effects of feedback interventions on everyday energy use have observed substantial variation in effect size, both within and between studies with explanations often as varied as the results themselves. The variation is partly due to demographic, housing, and climate characteristics of the households. Studies have found that households with higher income, higher education levels, and higher electricity use show greater reductions when feedback is provided [5,24-26]. Other research has argued a positive correlation between income and household consumption levels [27,28], although some have found that the link is not always clear (Brandon \& Lewis, 1999). In terms of income and consumption level reductions as a result of feedback (or other) interventions, again, researchers have found a positive correlation to exist $[5,28]$, while others have not [25]. Climate will also impact reductions in use, as the same type of house would have a different demand in a hot tropical or sub-tropical climate than it would in a cool, temperate climate. Households in more extreme climates (hotter in summer and colder in winter) would appear to have more potential for reducing electricity use. However, several studies have found that feedback has more of an impact when temperatures are more moderate $[24,29]$. Significant gaps remain on the effectiveness of feedback on different demographic groups and on households living in different climate regions.

Some studies have found that certain types of households respond better to feedback than others, but a considerable amount of uncertainty still exists over how different households will respond to increased level of information [30]. In a recent study of 21 households Wallenborn and colleagues [31] found that electricity feedback through smart meters could change electricity perception but only in households already interested or involved in energy savings or willing to understand the information provided. As a result of real-time feedback, the participants in another study reported taking action to reduce their energy consumption, however, the study found a statistically insignificant reduction in actual electricity consumption by the participants [22]. Alahmad and colleagues [22] suggest this could be due to the selfselection of participants and their already invested interest in electricity conservation prior to the study. In another recent study with 28 Australian households, Strengers [32] acknowledges in-home display feedback as an important visualisation tool illuminating what would otherwise be invisible. However, Strengers [32] argues that feedback has the potential to ignore practices considered non-negotiable and legitimise particular practices, for example, the routine use of clothes dryers by concentrating on what can be readily measured and saved rather than whether the practice is normal or necessary. Strengers [32] posits that failing to engage with the practices seen to be non-negotiable conditions of everyday living may cause householders to lose interest in this type of feedback over time. Indeed Ellegard and Palm [33] argue that a deeper knowledge of everyday energy consumption activities makes everyday life more sustainable.

Despite numerous studies on the effects of feedback, the potential impacts of feedback programs, especially large-scale, remain highly uncertain. In the majority of trials, feedback is designed and tested only in the context of its ability to facilitate a change in behaviour or to persuade consumers to use less power. While feedback provision generally results in consumers using less electricity for the period that it is installed, precisely why this is the case remains unclear [9,31]. As a result of testing feedback only for its energy saving potential, the scope of design and potential uses and interactions with regard to feedback, has been limited [34]. 


\section{Goal Setting}

One theme that is prevalent in the literature is the role of goal-setting with feedback. Several authors argue that feedback is only effective when it leads to the setting of a performance goal and that goal-setting is only effective in the presence of feedback that allows participants to evaluate their performance [35-38]. In Becker's [35] study, feedback alone led to a $4.5 \%$ decrease compared to results of energy savings of 5.7\% when feedback was combined with the modest goal of a $2 \%$ reduction and $15.1 \%$ in electricity savings when feedback was combined with the difficult goal of a $20 \%$ reduction in electricity. More recently, Schultz [39] detailed examples of the energy savings that such strategies can achieve, including an assessment of OPower's increasingly popular Home Energy Report program which has achieved savings as high as $8 \%$ for those households that set personal conservation goals. Bonino and colleagues [40] in their online study of nearly 1000 participants found that energy goal setting is better for improving energy consumption.

The contribution of goal-setting to feedback programs, however, is not straight-forward. There are, according to Fischer [12] in her review of feedback programs, many studies where feedback alone appears to have worked. Fischer [12] also cites other studies involving commitment that delivered a small result with one study actually finding no effect on energy consumption. One possible explanation is that goals can be both implicit and explicit [36]. Studies where feedback alone appears to have worked could be the result of implicitly made commitments for goals that participants find meaningful and try to achieve for themselves.

\section{Comparison Standards}

The two main types of comparisons that have been investigated in the literature are "historic" and "normative" [12]. Historic feedback refers to consumption reported relative to the consumption of the same household from a similar time period in the past. Normative comparative feedback refers to consumption of a household reported in comparison to the consumption of some other similar group of households.

\subsection{Historic Standards}

Historic feedback provides residents with some frame of reference for their consumption levels and is generally perceived to be effective in this regard. In one example where it was implemented for the first time, treatment groups showed a $10 \%$ decrease in consumption which was maintained for more than three years [Wilhite and colleagues cited in 41]. The historic standard was found to be much preferred to a normative standard by focus group participants in the UK [42]. It has also been found to be the most readily recalled piece of information on an energy bill and what customers use to try and understand their consumption patterns [43]. In their study, Kempton and Layne [44] found that only $41 \%$ of their participants paid attention to the recent addition on the energy bill of a historic comparison standard. Despite Kempton and Layne's findings, historic feedback appears to be readily understandable, relevant, and useful for consumers. In her review, Fischer [45] found that an historic standard was one of the main features of some of the most effective studies for overall conservation.

\subsection{Normative Standards}

The effectiveness of normative comparative feedback is unclear. Comparing the consumption of one household to that of others is said to elicit social pressure to understand why consumption levels differ and to stimulate competition and ambition [45,46]. Cialdini [47] identified the importance of social proof in human decision making as people tend to imitate behaviour of others. Indeed, there are reports in which consumers have indicated that this sort of comparison based on similar demographics would be of interest to them $[43,48]$. In their study, Kempton and Layne [44] found that $70 \%$ of their participants had at some time discussed their bills with other people, including their neighbours. More recently, participants of another study indicated their interest in sharing energy-consumption feedback with family and friends [49]. It has been suggested that neighbour-based comparisons may be meaningful as neighbours tend to report similar attitudes and behaviours [Beaman and Vaske cited in 50]. Indeed, the effect of peers has been found to be more effective than incentives such as saving money, conserving resources, or being socially conscious [51]. Whilst the highest quality comparison combines various household attributes it has also been suggested that for practicality, individual streets in groups of 30 addresses is a good basis for geographical comparison [50]. An additional benefit of this type of comparative feedback is that there is no need for weather-adjusting.

Comparative standards are not universally popular, however. A UK study reported findings from their focus groups which suggested normative comparative standards to be very unpopular [42]. This preference may be cultural [45]. American [43,46,51,52] and Norwegian [48] studies have found that residents like normative comparison standards. Allcott [52] reviewed data from randomised natural field experiments of 600,000 treatment and control households in the United States that employed comparative electricity-use feedback, tips for energy conservation and an injunctive message of smiley face/s. Allcott [52] found that the effect of the intervenetion was equivalent to that of a short-run electricity price 
increase of $11 \%$ to $20 \%$ and that the cost effectiveness of the intervention compared favourably to traditional energy conservation programs.

As mentioned above, the effect of comparative standards on actual energy conservation is less clear. Bittle and colleagues [24] found in their study a result opposite to what was intended. They found that those who received the comparative standard feedback consumed more than those who did not. In ten studies reviewed by Fischer [45], there was no savings benefit with comparative standard feedback. Fischer [45] postulates that savings achieved by high users who were encouraged through the comparison to conserve energy may have been cancelled out by lower than average users being inadvertently encouraged to increase energy use because of the comparative standard. This phenomenon has been referred to as the "boomerang" effect and demonstrated in a study undertaken by Schultz and colleagues [46]. In their study, all households received comparative electricity-use feedback in which they were compared to their neighbours, but one group also received an injunctive message in the form of a hand-written smiley-face for households whose consumption was below the average level, and a sad-face for those whose consumption was above the average level. They found that those who consumed less than the average, but received the injunctive message of encouragement (the smiley face), maintained low consumption, whereas, those lower than average consumers who did not receive the injunctive message, increased their consumption. This study demonstrated that the "boomerang" effect can be mediated by not only providing descriptive norms but also including injunctive norms that somehow indicate what is commonly socially acceptable (or unacceptable) within a certain culture [46].

\section{Criteria for Effective Feedback}

While research is ongoing into the most effective types of feedback for encouraging conservation, some trends have emerged in the literature. It has been suggested that feedback must meet three characteristics for optimum effectiveness [53]. These characteristics include that: feedback must be received as close in time to the consumption event as possible; be related to some standard; and be presented in such a way that is meaningful to the consumer [53]. A fourth characteristic of customised and personalised feedback for individual households has also been suggested by Darby [54] and McMakin and colleagues [55]. According to Fischer [45], feedback should also be computerised, interactive, have appliance specific breakdown of consumption use and be provided over a prolonged period of time. A smaller body of research has explored detailed specifics of what should be included in feedback and while interesting and somewhat informa- tive, Fischer [45] has argued that specific features may not always be generalizable across demographic groupings or cultures.

\subsection{Trusted and Credible Feedback Source}

The feedback information needs to be supplied by a trusted and credible source [19]. People can have an inherent distrust of social institutions and think that industry, business and government decisions and priorities are not aligned with energy efficiency objectives [56]. In an interesting study undertaken by Miller and Ford [57], they demonstrated this inherent distrust by sending a letter soliciting an energy conservation program using three different letterheads and they found that the letter that did not list affiliation with the utility received a significantly better response. In examining conservation programs, the use of community-based, non-profit contractors was effective $[56,58]$.

\subsection{Presentation of Energy Consumption Detail}

For reporting consumption relative to a historic standard, Roberts, Humphries, and Hyldon [42] and Fitzpatrick and Smith [59] found that most of their participants preferred a bar graph representation. For comparative standards, Egan and colleagues [43] found that customers preferred a horizontal "sliding scale" bar chart that indicated on the scale with an arrow where the home's consumption lied. This was preferred over a distribution chart mimicking a bell curve. In general, Egan and colleagues [43] found that the comprehension of the graphics was relatively low, but that adding end-point labels to the charts helped. In marked contrast to these findings are those of Iyer and colleagues [50] who found that the distribution chart was easily understood by participants. In another study, Wilhite and colleagues [48] found their focus group participants were divided over the preference for linear representation and distribution charts for depicting energy consumption. In her review of feedback literature, Fischer [45] summarized her findings by suggesting that, for historical comparisons, vertical bar charts were preferred and for comparative feedback, the single bar graph is preferred. For information displays in general, Roberts and Baker [60] found that graphical displays such as pie charts were preferred, and that they required text labels for improved clarity. It appears that appliance usage information is also best represented in pie chart format $[48,61]$.

\subsection{Appliance Usage Charts}

Often consumers believe that the appliances that are most visible to them (e.g. lights, dishwashers) are the ones that consume the most electricity [48]. It has been argued, therefore, that providing information on specific appli- 
ances and the home's appliance mix is desirable and beneficial to customers and to electricity conservation [59,61]. In her review, Fischer [45] found that some of the most effective studies often contained appliance specific detail. However, appliance-monitoring systems are expensive and require user configuration [49]. Sundramoorthy and colleagues [49] found in their study that participants were able to attribute dips and curves in the load to particular appliances and activities thereby possibly negating the need and therefore the cost of specific appliance-monitoring.

\subsection{Consumption Metrics}

The electricity consumption metric is also an important consideration. Dollar values of consumption are considered by some authors to be more desirable and useful to consumers [12,13]. Farhar and Fitzpatrick [25] found that their participants liked cost-based energy feedback and that it consistently resulted in reductions. However, Hutton and colleagues [62] and Fitzpatrick and Smith [59] found that feedback emphasising financial values did not have positive results across all their samples.

Environmental metrics have been used infrequently by researchers with Fischer [45] reporting only two in her review of feedback studies. The use of environmental metrics is perhaps one way of stimulating personal norms with regard to environmental concern $[12,59]$ especially given current climate change issues. In their small study, Brandon and Lewis [25] found no significant impact on electricity conservation with the use of feedback containing environmental metrics. The link between environmental concerns and consumption may not always be obvious even to environmentally aware households [27].

\subsection{Conservation Tips-Customising}

Customisation seems to be important for conservation tips or advice to be effective [49]. In one study a customised newsletter including conservation tips was distributed with presumably customised consumption information and customers reported that the tips were the most useful in helping them to conserve [61]. Other focus group research recorded participants' dislike for a generic leaflet that would have been provided as an insert and their intention to discard such an insert [42].

\subsection{Frequency}

More immediate and frequent feedback is more likely to result in behaviour change [63]. Allen and Janda's [64] review of feedback studies recognised the primary benefit of real-time feedback as that of affecting customer awareness. The current state of the art for feedback devices is electric monitors that indicate how much electricity the household is using at any given moment. Elec- tric monitors have the advantage over written feedback of being completely automated and likely being much more cost effective on a large-scale basis. While pilot projects testing continuous feedback have been more common recently [18], continuous feedback has been the subject of research since the 1970s. McClelland and Cook [65] was the first continuous feedback study and it found savings of 12 percent.

Ueno and colleagues [66,67] have conducted studies in Japan using continuous energy monitors. The 2005 study considered meters which disaggregated feedback by appliance and achieved savings of 17.8 percent. The 2006 study achieved savings of 9 percent with meters that did not disaggregate by appliance. Allen and Janda [64] reported on a study of 10 households that found no conservation effect from a continuous feedback device known as "The Energy Detective” (TED). The study participants reported that TED was not user-friendly and this seemed to cause the participants to ignore the device rather than explore and use the manual. Interestingly, in a more recent study with TED, Parker and colleagues [67] identified average savings among 17 households of 7.4 percent. However, the savings in the study ranged widely and the study participants were self-selected.

\subsection{Delivery Medium}

Fischer [45] found that the common feature in the "best" of 10 feedback studies she reviewed was interactivity in a computerised format and it could be argued that feedback delivery via email is an extension of this $[13,68]$. Gleerup and colleagues [68] in a recent Danish study found that timely information about a household's exceptional consumption communicated via email and sms messaging resulted in average reductions in total annual electricity use of about 3\%. They argued that the type of feedback tested in their study could have a larger effect in other countries because Danish households are likely to be more efficient with electricity consumption as Denmark has the highest marginal electricity price in the world [68]. Email delivery also allows for feedback to be sent directly to the consumer and it can easily be linked to websites that are perhaps more interactive than the email feedback alone. Fischer [45] identified that effective feedback allows for multiple options that the user can choose interactively which is possible with internetbased feedback.

While email and internet-based feedback is generally more feasible for utilities [61] and there are some studies indicating that customers perceive paper-based feedback as wasteful [60], demographics and connectivity factors need to be considered as well. Martinez and Geltz [61] found in their study of 400 Californian residential customers that two-thirds preferred paper-based mail as their choice of medium for feedback, with a similar percentage 
of commercial customers indicating the same preference.

\subsection{Layout, Appearance and Location}

Fitzpatrick and Smith [59], Donnelly [69], Hargreaves et al. [9], Riche et al. [70], Karjalainen [34] Rodgers and Bartram [71] and Bonino and colleagues [40] explore design issues related to the integration of feedback into the household. Concerns over placement, aesthetic appeal and privacy considerations are found to be important considerations in the successful and long-term integration of a feedback medium in the home [70]. Bonino and colleagues [40] found that power visualisation should be in every room or on a portable device, e.g. a smart phone and that colour-based feedback was more easily understood and appreciated by their participants. Ambient and artistic visualisation was found to be a promising method of providing real-time feedback of residential energy use [71]. Preferences regarding functionality and aesthetic appeal vary widely both between and within households however, with results suggesting that regardless of the functionality of the feedback, devices which are aesthetically displeasing tended to become hidden from view and not utilised [9]. Confusingly, half of the participants in the Bonino and colleagues' study [40], wanted a less central "aesthetically acceptable" location while the other half wanted a visible location to track electricity use.

\section{Conclusions}

In general, the literature finds that feedback can reduce electricity consumption in homes by 5 to 20 percent [13], and that it works best when it is:

- delivered regularly;

- presented plainly and engagingly;

- tailored to the householder;

- interactive and digital;

- capable of providing information by appliance;

- accompanied by advice for reducing electricity use, and

- associated with a challenging goal for energy conservation.

However, there are key uncertainties from the literature and significant gaps still remain in our knowledge of the effectiveness and cost benefit of feedback. A number of research gaps identified by EPRI [18] and verified in this review include:

- The effect of feedback on consumers in different demographic groups;

- The effect of feedback on appliance purchasing decisions;

- The response effect on energy consumption from different formats of feedback;

- Whether feedback continues to work over time or whether it needs to be renewed/reshaped to keep householders engaged and maintain any conservation effects.

In addition, further gaps in research have been highlighted through this review and they include:

- The ability for feedback to facilitate the sharing of electricity information between households, friends or neighbours is almost entirely unexplored;

- The scope of design, potential uses and interactions with regard to feedback has been limited;

- The divergence of cost-benefit calculations for feedback with advanced metering infrastructure needs to be explored as does the conditions under which the costs of feedback outweigh the benefits.

This review has explored one potential solution, more detailed feedback, to help control the growth of residential energy and the expansion of electricity infrastructure. Finding such solutions could become increasingly important if demand for heating and cooling appliances continues at its current projection and/or hybrid and fullelectric vehicles become a substantial portion of automobile sales. These are just two examples of situations which would add significant demand to the grid and where it would become more critical to try and control how and when consumers used their heating and cooling appliances and recharged their cars so as not to exceed peak capacity. Research shows that feedback does have the potential to positively affect residential electricity conservation. With increasing smart grid investment and improving feedback devices to be more user-friendly there will be greater opportunity to connect the consumer to the grid and therefore study the effect of feedback on large groups of consumers. Results from such studies would potentially be of interest to a diverse range of professional areas such as social science, computer science, power engineering and energy economics.

\section{REFERENCES}

[1] P. Simshauser, E. Molyneux and M. Shepherd, "The Entry Cost Shock and the Re-Rating of Power Prices in New South Wales, Australia," Australian Economic Review, Vol. 43, No. 2, 2010, pp. 114-135.

doi:10.1111/j.1467-8462.2010.00584.x

[2] A. Faruqui, S. Sergici and A. Sharif, "The Impact of Informational Feedback on Energy Consumption-A Survey of the Experimental Evidence,” Energy, Vol. 35, No. 4, 2010, pp. 1598-1608. doi:10.1016/j.energy.2009.07.042

[3] Australian Energy Market Commission, "Retail Electricity Price Estimates: Final Report for 2010-2011 to 20132014,” Australian Energy Market Commission, Sydney, 2011.

[4] R. A. Winett, J. H. Kagel, R. C. Battalio and R. C. Winkler, "Effects of Monetary Rebates, Feedback, and Information on Residential Electricity Conservation,” Journal of Applied Psychology, Vol. 63, No. 1, 1978, pp. 73-80. 
doi:10.1037/0021-9010.63.1.73

[5] H. Wilhite and R. Ling, "Measured Energy Savings from a More Informative Energy Bill," Energy and Buildings, Vol. 22, No. 2, 1995, pp. 145-155.

doi:10.1016/0378-7788(94)00912-4

[6] L. Nielsen, "How to Get the Birds in the Bush into Your Hand: Results from a Danish Research Project on Electricity Savings,” Energy Policy, Vol. 21, No. 11, 1993, pp. 1133-1144. doi:10.1016/0301-4215(93)90263-F

[7] T. Ueno, R. Inada, O. Saeki and K. Tsuji, "Effectiveness of an Energy-Consumption Information System for Residential Buildings,” Applied Energy, Vol. 83, No. 8, 2006, pp. 868-883. doi:10.1016/j.apenergy.2005.09.004

[8] A. Gronhoj and J. Thogersen, "Feedback on Household Electricity Consumption: Learning and Social Influence Processes," International Journal of Consumer Studies, Vol. 35, No. 2, 2011, pp. 138-145. doi:10.1111/j.1470-6431.2010.00967.x

[9] T. Hargreaves, M. Nye and J. Burgess, "Making Energy Visible: A Qualitative Field Study of How Householders Interact with Feedback from Smart Energy Monitors," Energy Policy, Vol. 38, No. 10, 2010, pp. 6111-6119. doi:10.1016/j.enpol.2010.05.068

[10] S. Darby, "Smart Metering: What Potential for Householder Engagement?” Building Research and Information, Vol. 38, No. 5, 2010, pp. 442-457. doi:10.1080/09613218.2010.492660

[11] M. Martiskainen and J. Coburn, “The Role of Information and Communication Technologies (ICTs) in Household Energy Consumption-Prospects for the UK," Energy Efficiency, Vol. 4, No. 2, 2011, pp. 209-221. doi:10.1007/s12053-010-9094-2

[12] C. Fischer, "Feedback on Household Electricity Consumption: A Tool for Saving Energy?” Energy Efficiency, Vol. 1, No. 1, 2008, pp. 79-104. doi:10.1007/s12053-008-9009-7

[13] S. Darby, "The Effectiveness of Feedback on Energy Consumption: A Review for DEFRA of the Literature on Metering, Billing and Direct Displays,” Environmental Change Institute, University of Oxford, Oxford, 2006.

[14] G. Wood and M. Newborough, "Dynamic Energy-Consumption Indicators for Domestic Appliances: Environment, Behaviour and Design,” Energy and Buildings, Vol. 35, No. 8, 2003, pp. 821-841. doi:10.1016/S0378-7788(02)00241-4

[15] G. Jacucci, A. Spagnolli, L. Gamberini, A. Chalambalakis, C. Björksog, M. Bertoncini, C. Torstensson and P. Monti, "Designing Effective Feedback of Electricity Consumption for Mobile User Interfaces,” PsychNology Journal, Vol. 7, No. 3, 2009, pp. 265-289.

[16] J. H. van Houwelingen and W. F. van Raaij, “The Effect of Goal-Setting and Daily Electronic Feedback on InHome Energy Use,” Journal of Consumer Research, Vol. 16, No. 1, 1989, pp. 98-105. doi:10.1086/209197

[17] E. Kamenica, S. Mullainathan and R. Thaler, "Helping Consumers Know Themselves," American Economic Review, Vol. 101, No. 3, 2011, pp. 417-422. doi:10.1257/aer.101.3.417
[18] Electric Power Research Institute, "Residential Electricity Use Feedback: A Research Synthesis and Economic Framework 1016844,” Electric Power Research Institute, Palo Alto, 2009.

[19] J. Burgess and M. Nye, "Re-Materialising Energy Use through Transparent Monitoring Systems,” Energy Policy, Vol. 36, No. 12, 2008, p. 4454. doi:10.1016/j.enpol.2008.09.039

[20] A. Spagnolli, N. Corradi, L. Gamberini, E. Hoggan, G. Jacucci, C. Katzeff, L. Broms and L. Jonsson, "Eco-Feedback on the Go: Motivating Energy Awareness,” Computer, Vol. 44, No. 5, 2011, pp. 38-45. doi:10.1109/MC.2011.125

[21] D. J. Kerrigan, L. Gamberini, A. Spagnolli and G. Jacucci, "Smart Meters: A Users' View," PsychNology Journal, Vol. 9, No. 1, 2011, pp. 55-72.

[22] M. A. Alahmad, P. G. Wheeler, A. Schwer, J. Eiden and A. Brumbaugh, "A Comparative Study of Three Feedback Devices for Residential Real-Time Energy Monitoring," IEEE Transactions on Industrial Electronics, Vol. 59, No. 4, 2012, pp. 2002-2013. doi:10.1109/TIE.2011.2165456

[23] S. S. van Dam, C. A. Bakker and J. D. M. van Hal, "Home Energy Monitors: Impact over the Medium-Term,” Building Research and Information, Vol. 38, No. 5, 2010, pp. 458-469. doi:10.1080/09613218.2010.494832

[24] R. G. Bittle, R. Valesano and G. Thaler, "The Effects of Daily Cost Feedback on Residential Electric Consumption,” Behaviour Modification, Vol. 3, No. 2, 1979, pp. 187-202. doi:10.1177/014544557932004

[25] G. Brandon and A. Lewis, "Reducing Household Energy Consumption: A Qualitative and Quantitative Field Study," Journal of Environmental Psychology, Vol. 19, No. 1, 1999, pp. 75-85. doi:10.1006/jevp.1998.0105

[26] I. Ayres, S. Raseman and A. Shih, "Evidence from Two Large Field Experiments that Peer Comparison Feedback can Reduce Residential Energy Usage,” Rochester, 2009.

[27] B. Gatersleben, L. Steg and C. Vlek, "Measurement and Determinants of Environmentally Significant Consumer Behavior," Environment and Behavior, Vol. 34, No. 3, 2002, pp. 335-362. doi:10.1177/0013916502034003004

[28] D. A. Guerin, B. L. Yust and J. G. Coopet, “Occupant Predictors of Household Energy Behavior and Consumption Change as Found in Energy Studies Since 1975," Family and Consumer Sciences Research Journal, Vol. 29, No. 1, 2000, pp. 48-80. doi:10.1177/1077727X00291003

[29] D. C. Mountain, "Real-Time Feedback and Residential Electricity Consumption: British Columbia and Newfoundland and Labrador Pilots,” 2007.

[30] K. Ehrhardt-Martinez, J. A. S. Laitner and K. A. Donnelly, "Chapter 10-Beyond the Meter: Enabling Better Home Energy Management,” In: S. Fereidoon Perry, Ed., Energy, Sustainability and the Environment, ButterworthHeinemann, Boston, 2011, pp. 273-303.

[31] G. Wallenborn, M. Orsini and J. Vanhaverbeke, "Household Appropriation of Electricity Monitors," International Journal of Consumer Studies, Vol. 35, No. 2, 2011, 
pp. 146-152. doi:10.1111/j.1470-6431.2010.00985.x

[32] Y. Strengers, "Negotiating Everyday Life: The Role of Energy and Water Consumption Feedback," Journal of Consumer Culture, Vol. 11, No. 3, 2011, pp. 319-338. doi:10.1177/1469540511417994

[33] K. Ellegård and J. Palm, "Visualizing Energy Consumption Activities as a Tool for Making Everyday Life More Sustainable," Applied Energy, Vol. 88, No. 5, 2011, pp. 1920-1926. doi:10.1016/j.apenergy.2010.11.019

[34] S. Karjalainen, "Consumer Preferences for Feedback on Household Electricity Consumption," Energy and Buildings, Vol. 43, No. 2-3, 2011, pp. 458-467. doi:10.1016/j.enbuild.2010.10.010

[35] L. J. Becker, “Joint Effect of Feedback and Goal Setting on Performance: A Field Study of Residential Energy Conservation,” Journal of Applied Psychology, Vol. 63, No. 4, 1978, pp. 428-433. doi:10.1037/0021-9010.63.4.428

[36] C. Seligman, L. J. Becker and J. M. Darley, "Encouraging Residential Energy Conservation through Feedback," Advances in Environmental Psychology, Vol. 3, 1981, pp. 93-113.

[37] L. T. McCalley and C. J. H. Midden, "Energy Conservation through Product-Integrated Feedback: The Roles of Goal-Setting and Social Orientation,” Journal of Economic Psychology, Vol. 23, No. 5, 2002, pp. 589-603. doi:10.1016/S0167-4870(02)00119-8

[38] L. T. McCalley and C. J. H. Midden, "Making Energy Feedback Work,” In: P.-P. Verbeek and A. Slob, Eds., User Behavior and Technology Development, Springer Netherlands, Vol. 20, 2006, pp. 127-137. doi:10.1007/978-1-4020-5196-8_13

[39] P. W. Schultz, "Making Energy Conservation the Norm," In: K. Ehrhardt-Martinez and J. A. S. Laitner, Eds., People-Centered Initiatives for Increasing Energy Savings, ACEEE, Colorado, 2010.

[40] D. Bonino, F. Corno and L. De Russis, "Home Energy Consumption Feedback: A User Survey," Energy and Buildings, Vol. 47, 2012, pp. 383-393. doi:10.1016/j.enbuild.2011.12.017

[41] G. Wood and M. Newborough, "Energy-Use Information Transfer for Intelligent Homes: Enabling Energy Conservation with Central and Local Displays,” Vol. 39, No. 4, 2007, pp. 495-503. doi:10.1016/j.enbuild.2006.06.009

[42] S. Roberts, H. Humphries and V. Hyldon, "Consumer Preferences for Improving Energy Consumption Feedback: Report to Ofgem,” Centre for Sustainable Energy, Bristol, 2004.

[43] C. Egan, W. Kempton, A. Eide, D. Lord and C. Payne, "How Customers Interpret and Use Comparative Graphics of Their Energy Use,” ACEEE, Washington D.C., 1996, pp. 39-46.

[44] W. Kempton and L. L. Layne, "The Consumer's Energy Analysis Environment,” Energy Policy, Vol. 22, No. 10, 1994, pp. 857-866. doi:10.1016/0301-4215(94)90145-7

[45] C. Fischer, "Discussion Paper 8: Influencing Electricity Consumption Via Consumer Feedback: A Review of Experience,” ECEEE 2007 Summer Study, Berlin, 2007.
[46] P. W. Schultz, J. M. Nolan, R. B. Cialdini, N. J. Goldstein and V. Griskevicius, "The Constructive, Destructive, and Reconstructive Power of Social Norms,” Psychological Science, Vol. 18, No. 5, 2007, pp. 429-434. doi:10.1111/j.1467-9280.2007.01917.x

[47] R. B. Cialdini, "Influence: The Psychology of Persuasion,” William Morrow \& Co., New York, 1993.

[48] H. Wilhite, A. Hoivik and J. Olsen, "In Advances in the Use of Consumption Feedback Information in Energy Billing: The Experiences of a Norwegian Utility," ECEEE 1999 Summer Study Proceedings, 1999.

[49] V. Sundramoorthy, G. Cooper, N. Linge and L. Qi, “Domesticating Energy-Monitoring Systems: Challenges and Design Concerns," IEEE on Pervasive Computing, Vol. 10, No. 1, 2011, pp. 20-27. doi:10.1109/MPRV.2010.73

[50] M. Iyer, W. Kempton and C. Payne, "Comparison Groups on Bills: Automated, Personalized Energy Information," Energy and Buildings, Vol. 38, No. 8, 2006, pp. 988-996. doi:10.1016/j.enbuild.2005.11.009

[51] J. M. Nolan, P. W. Schultz, R. B. Cialdini, N. J. Goldstein and V. Griskevicius, "Normative Social Influence Is Underdetected,” Personality and Social Psychology Bulletin, Vol. 34, No. 7, 2008, pp. 913-923. doi:10.1177/0146167208316691

[52] H. Allcott, "Social Norms and Energy Conservation," Journal of Public Economics, Vol. 95, No. 9-10, 2011, pp. 1082-1095. doi:10.1016/j.jpubeco.2011.03.003

[53] C. J. H. Midden, J. E. Meter, M. H. Weenig and H. J. A. Zieverink, "Using Feedback, Reinforcement and Information to Reduce Energy Consumption in Households: A Field-Experiment,” Journal of Economic Psychology, Vol. 3, No. 1, 1983, pp. 65-86. doi:10.1016/0167-4870(83)90058-2

[54] S. Darby, "Making It Obvious: Designing Feedback into Energy Consumption,” In Second International Conference on Energy Efficiency in Household Appliances and Lighting, Naples, 2000.

[55] A. H. McMakin, E. L. Malone and R. E. Lundgren, "Motivating Residents to Conserve Energy without Financial Incentives," Environment and Behavior, Vol. 34, No. 6, 2002, pp. 848-863. doi:10.1177/001391602237252

[56] L. Lutzenhiser, "Social and Behavioral Aspects of Energy use," Annual Review of Energy and the Environment, Vol. 18, No. 1, 1993, pp. 247-289. doi:10.1146/annurev.eg.18.110193.001335

[57] R. D. Miller and J. M. Ford, "Shared Savings in the Residential Market: A Public/Private Partnership for Energy Conservation Urban Consortium for Technology Initiatives,” Energy Task Force, Baltimore, 1985.

[58] R. B. Cialdini, "Basic Social Influence Is Underestimated,” Psychological Inquiry, Vol. 16, No. 4, 2005, pp. 158-161. doi:10.1207/s15327965pli1604_03

[59] G. Fitzpatrick and G. Smith, “Technology-Enabled Feedback on Domestic Energy Consumption: Articulating a Set of Design Concerns,” IEEE on Pervasive Computing, Vol. 8, No. 1, 2009, pp. 37-44. doi:10.1109/MPRV.2009.17

[60] S. Roberts and W. Baker, "Towards Effective Energy 
Information. Improving Consumer Feedback on Energy Consumption. A Report to OFGEM,” Centre for Sustainable Energy, Bristol, 2003.

[61] M. S. Martinez and C. R. Geltz, "Utilizing a Pre-Attentive Technology for Modifying Customer Energy Usage European Council for an Energy-Efficient Economy," 2005.

[62] R. B. Hutton, G. A. Mauser, P. Filiatrault and O. T. Ahtola, "Effects of Cost-Related Feedback on Consumer Knowledge and Consumption Behavior: A Field Experimental Approach,” Journal of Consumer Research, Vol. 13, No. 3, 1986, pp. 327-336. doi:10.1086/209072

[63] M. J. Bekker, T. D. Cumming, N. K. P. Osborne, A. M. Bruining, J. I. McClean and L. S. Leland Jr., "Encouraging Electricity Savings in a University Residential Hall through a Combination of Feedback, Visual Prompts, and Incentives," Journal of Applied Behavior Analysis, Vol. 43, No. 2, 2010, pp. 327-331. doi:10.1901/jaba.2010.43-327

[64] D. Allen and K. B. Janda, "In the Effects of Household Characteristics and Energy Use Consciousness on the Effectiveness of Real-Time Energy Use Feedback: A Pilot Study American Council for an Energy-Efficient Economy 2006 Summer Study, Washington D.C., 2006,” ACEEE: Washington D.C., 2006.

[65] W. Abrahamse, L. Steg, C. Vlek and T. Rothengatter, “A Review of Intervention Studies Aimed at Household Energy Conservation,” Journal of Environmental Psychology, Vol. 25, No. 3, 2005, pp. 273-291. doi:10.1016/j.jenvp.2005.08.002

[66] T. Ueno, R. Inada, O. Saeki and K. Tsuji, "In Effectiveness of Displaying Energy Consumption Data in Residen- tial Houses: Analysis on How the Residents Respond, 2005 Summer Study of the European Council for an Energy Efficient Economy, Stockholm, 2005,” ECEEE, Stockholm, 2005, pp. 1289-1299.

[67] D. Parker, D. Hoak and J. Cummings, "Pilot Evaluation of Energy Savings and Persistence from Residential Energy Demand Feedback Devices in a Hot Climate. In Summer Study on Energy Efficiency in Buildings-The Climate for Efficiency is Now," ACEEE, Pacific Grove, CA, 2010.

[68] M. Gleerup, A. Larsen, S. Leth-Petersen and M. Togeby, "The Effect of Feedback by Text Message (SMS) and Email on Household Electricity Consumption: Experimental Evidence,” The Energy Journal, Vol. 31, No. 3, 2010, pp. 113-132. doi:10.5547/ISSN0195-6574-EJ-Vol31-No3-6

[69] K. A. Donnelly, "The Technological and Human Dimensions of Residential Feedback: An Introduction to the Broad Range of Today's Feedback Strategies,” In: K. Ehrhardt-Martinez and J. A. S. Laitner, Eds., PeopleCentered Initiatives for Increasing Energy Savings, ACEEE, Colorado, 2010.

[70] Y. Riche, J. Dodge and R. A. Metoyer, "Studying Always-On Electricity Feedback in the Home," Proceedings of the SIGCHI Conference on Human Factors in Computing Systems, ACM, Atlanta, 2010, pp. 1995-1998.

[71] J. Rodgers and L. Bartram, "Exploring Ambient and Artistic Visualization for Residential Energy Use Feedback," IEEE Transactions on Visualization and Computer Graphics, Vol. 17, No. 12, 2011, pp. 2489-2497. doi:10.1109/TVCG.2011.196 